\title{
Does Variant Luteinizing Hormone (V-LH) Predispose to Improper Testicular Position in Late Pregnancy?
}

\author{
MARKO KALEVA, HELENA VIRTANEN, ANNE-MAARIT HAAVISTO, KATHARINA MAIN, \\ NIELS E. SKAKKEBÆK, ILPO HUHTANIEMI, KERTTU IRJALA, AND JORMA TOPPARI
}

Department of Physiology [M.K., H.V., A.-M.H., I.H., J.T.], Department of Pediatrics [M.K., H.V., A.-M.H., J.T.], Department of Clinical Chemistry [K.I.], University of Turku, FIN-20520 Turku, Finland, Department of Growth and Reproduction [K.M., N.E.S.], Rigshospitalet, DK-2100 Copenhagen, Denmark, Institute of Reproductive and Developmental Biology [I.H.], Imperial College London, Hammersmith Campus, London W12 ONN, UK

\begin{abstract}
Undescended testes are a common urogenital malformation affecting 2-9\% of newborn boys. The etiology of cryptorchidism is probably heterogeneous, but insufficient androgen effect has been recognized as one cause of the condition. A common genetic variant $(\mathrm{V})$ form of $\mathrm{LH}$ occurs in apparently healthy individuals universally. Compared with wild-type (WT) LH, the V-LH molecule has increased bioactivity in vitro but shorter half-life in vivo. In the present study, we screened 93 cryptorchid (59 uni- and 34 bilateral) and 211 healthy boys for the occurrence of V-LH to evaluate whether it is related to testicular descent. Two immunofluorometric assays with different combinations of $\mathrm{MAb}$, one detecting WT-LH, the other detecting both WT- and $\mathrm{V}$-LH, were used to measure LH concentrations. The ratio of two LH measurements was used to assess the V-LH status. The prevalence of V-LH was similar in the control and cryptorchid groups, and the total prevalence of V-LH corresponded well to
\end{abstract}

\section{ABSTRACT}

the prevalence of V-LH in general Finnish population. Among cryptorchid boys, the prevalence of V-LH was dependent on gestational age: $6.7 \%$ at $\mathrm{GA}<37,20.9 \%$ at GA $37-39$, and $42.9 \%$ at GA of $40-42 \mathrm{wk}$. In contrast, the percentage of V-LH status was similar at different gestational ages in all control groups. We conclude that V-LH is not critical for normal testicular descent but the increased prevalence of V-LH among cryptorchid boys with GA $>40$, suggests that the lower hormonal efficacy of V-LH predisposes for improper testicular descent in late pregnancy. (Pediatr Res 58: 447-450, 2005)
Abbreviations
V-LH, variant luteinizing hormone
WT-LH, wild-type luteinizing hormone
GA, gestational age
MAb, monoclonal antibody

Undescended testes, i.e. cryptorchidism, is a common congenital malformation affecting 2-9\% of boys at birth and 1-3\% at 3 mo of age $(1,2)$. In most cases, the etiology of cryptorchidism remains unknown. Gonadotropin deficiency of cryptorchid boys has been found in several studies (3-5), and, since the 1930s, hormones have been used for treatment of undescended testes with varying success (6). Both serum gonadotropin and steroid hormone levels are high at the age of 1-3 mo and they decline to the childhood range by $4-6$ mo of age $(7,8)$. A high

Received August 5, 2004; accepted December 8, 2004.

Correspondence: Marko Kaleva, M.D., Department of Physiology, University of Turku, Kiinamyllynkatu 10, FIN-20520 Turku, Finland; e-mail: marko.kaleva@utu.fi

This study was funded by the Turku University Central Hospital, the Academy of Finland, the Nordic Academy for Advanced Study, and the European Commission under the framework of the "Quality of Life and Management of Living Resources" program (contract \#: BMH4-CT96-0314, QLK4-CT1999-01422, QLK4-2001-00269 and QLK42002-0063).

DOI: 10.1203/01.pdr.0000176918.68539.b4 percentage of cryptorchidism resolves spontaneously and almost all spontaneous descents are seen during the period of high hormone levels.

Variant LH is a universal polymorphism with highly varying frequency in different populations. The frequency varies from $0 \%$ in Kotas of Western India to $52 \%$ in Aboriginal Australians (9); in the Finnish population, the frequency of the V-LH is $27.7 \%$ (10). V-LH is a genetic variant of LH with two point mutations in its $\beta$-subunit gene. Both of these point mutations cause an amino acid change: $\operatorname{Trp}^{8} \mathrm{Arg}^{8}$ and $\mathrm{Ile}^{15} \mathrm{Thr}^{15}$ (11). In addition, the latter mutation introduces an extra glycosylation signal (Asn-X-Thr) into the LH $\beta$-chain (12). Due to these mutations, V-LH has different immunologic and biologic properties. The structural alterations change a specific antigene epitope present only in the $\mathrm{LH} \alpha / \beta$ - dimer, and consequently the MAb recognizing this epitope are unable to detect V-LH (13). Except for the immunologic changes, the extra sulfated oligosaccharide chain of $\mathrm{V}-\mathrm{LH}$ reduces its circulatory half 
time. As a compensatory mechanism, the V-LH $\beta$ promoter carries eight point mutations, and the activity of mutant $\mathrm{LH} \beta$ promoter is about $40 \%$ higher than that of the wild-type gene (14). Moreover, the intrinsic in vitro bioactivity of V-LH is on average $30 \%$ higher than that of wild-type LH. Nevertheless, because of its faster rate of elimination, V-LH apparently represents a biologically less active form of LH (9). Due to these facts, the variation in LH activity in the perinatal period could theoretically influence the incidence of cryptorchidism.

\section{METHODS}

The subjects consist of 304 sons of volunteer mothers who participated in our prospective cryptorchidism study in the Turku area in Finland. The volunteer mothers were recruited from local maternity clinics by public health nurses and all the boys were born in Turku University Central Hospital during the years 1997-2000. Clinical examinations were performed for the first time during the first $3 \mathrm{~d}$ after birth and again at the age of $3 \mathrm{mo}$. Midwives collected cord blood at birth and the samples were used for serum hCG and progesterone measurements. Another set of blood samples were collected at the 3-mo examination (age $3 \mathrm{mo} \pm 10 \mathrm{~d}$ ) between 1200 and $1800 \mathrm{~h}$. EMLA was used as a local anesthetic and $4 \mathrm{~mL}$ of peripheral venous blood were collected by an open needle method. The blood samples were allowed to clot and centrifuged, and the sera were separated and stored at $-20^{\circ} \mathrm{C}$ until analyzed for $\mathrm{LH}$.

Altogether, 93 cryptorchid and 211 healthy control boys of volunteer mothers were screened for the occurrence of V-LH. Out of 93 cryptorchid boys, 25 had high scrotal, 21 suprascrotal, 29 inguinal, and 18 nonpalpable testes at birth. In case of bilateral cryptorchidism, the most severe diagnosis was used for classification. Cryptorchidism was bilateral in 34 cases and unilateral in 59 cases ( 35 right and 24 left). All boys with retractile testes were carefully excluded from this study. The boys were grouped by gestational age (GA) into three groups: premature boys $(\mathrm{GA}<37)$, boys born after short pregnancy (GA 37-39), and boys born after long pregnancy (GA 40-42). Progesterone (87 cryptorchid and 204 controls) and hCG (88 cryptorchid and 204 controls) were analyzed from the cord blood serum samples. Due to the low total number of boys homozygous for $\mathrm{V}-\mathrm{LH}$, we combined them with boys heterozygous for V-LH in all analyses.

Immunoreactive LH concentrations were measured by two IFMA with different combinations of MAb. The reference method (Delfia hLH Spec kit, Wallac Oy, Turku, Finland), with two LH $\beta$-specific MAb, recognizes both forms of LH (assay 2). In the method to intact LH (I3/A2), the capture MAb recognizes an epitope in the intact $\mathrm{LH} \alpha / \beta$-dimer and the detection $\mathrm{MAb}$ recognizes the $\alpha$-subunit. This intact method (assay 1 ) recognizes only WT-
LH. The ratio of the two LH measurements (assay 1/assay 2) was used to assess the V-LH status. All the samples were categorized into three groups: 1) $>0.9$ (normal ratio, homozygous for WT-LH); 2) 0.15-0.9 (low ratio, heterozygous for $\mathrm{V}-\mathrm{LH}$ ); and 3 ) $<0.15$ ( 0 ratio, homozygous for $\mathrm{V}-\mathrm{LH}$ ). The sensitivity of the two IFMA assays was $0.05 \mathrm{IU} / \mathrm{L}$, and the intra- and interassay coefficients of variation were less than $5 \%$ and $10 \%$, respectively. AutoDELFIA (Wallac) was used for hCG measurements and the SPECTRIA Progesterone RIA test (Orion, Espoo, Finland) for progesterone measurements.

The study was approved by the local ethics committee and conducted according to the Helsinki II declaration. Written informed consent was given by the parents. Pearson $\chi^{2}$ test and logistic regression analysis were used for statistical analyses, which were performed with the SAS System for Windows release 8.2 (SAS Institute, Cary, NC). $p$ Values, odds ratios (OR), and 95\% confidence intervals $(95 \% \mathrm{CI})$ are used to indicate statistical significance.

\section{RESULTS}

Altogether, 304 boys, 93 cryptorchid and 211 healthy controls, were screened for the occurrence of V-LH. Out of the 304 boys, 80 (26.3\%) were either homo- or heterozygous for V-LH. The prevalence of V-LH was $26.1 \%$ (23.2\% heterozygous and $2.8 \%$ homozygous) in the control and $26.9 \%$ (20.4\% heterozygous and $6.5 \%$ homozygous) in the cryptorchid group. In the control group, V-LH was as common in all gestational age groups (Fig. $1 \mathrm{~A}$ ). However, among the cryptorchid boys, the prevalence of V-LH increased steadily with gestational age: $6.7 \%$ in the group of premature subjects, $20.9 \%$ in the group of $37-39$-wk pregnancies, and $42.9 \%$ in the group of $>40 \mathrm{wk}$ pregnancies (Fig. 1B).

Altogether, 42 cases (45.2\%) resolved the cryptorchidism spontaneously during the first $30 \mathrm{mo}$ of life. The spontaneous descent was as common in the V-LH as in the WT-LH groups, $48.0 \%$ and $44.1 \%$, respectively. The frequency of V-LH was similar in the uni- and bilaterally cryptorchid groups $(27.1 \%$ and $26.5 \%$ ) at birth.

Progesterone and hCG were measured from the cord blood samples. Generally, premature babies used to have higher hCG levels than full-term babies, and cryptorchid boys used to have
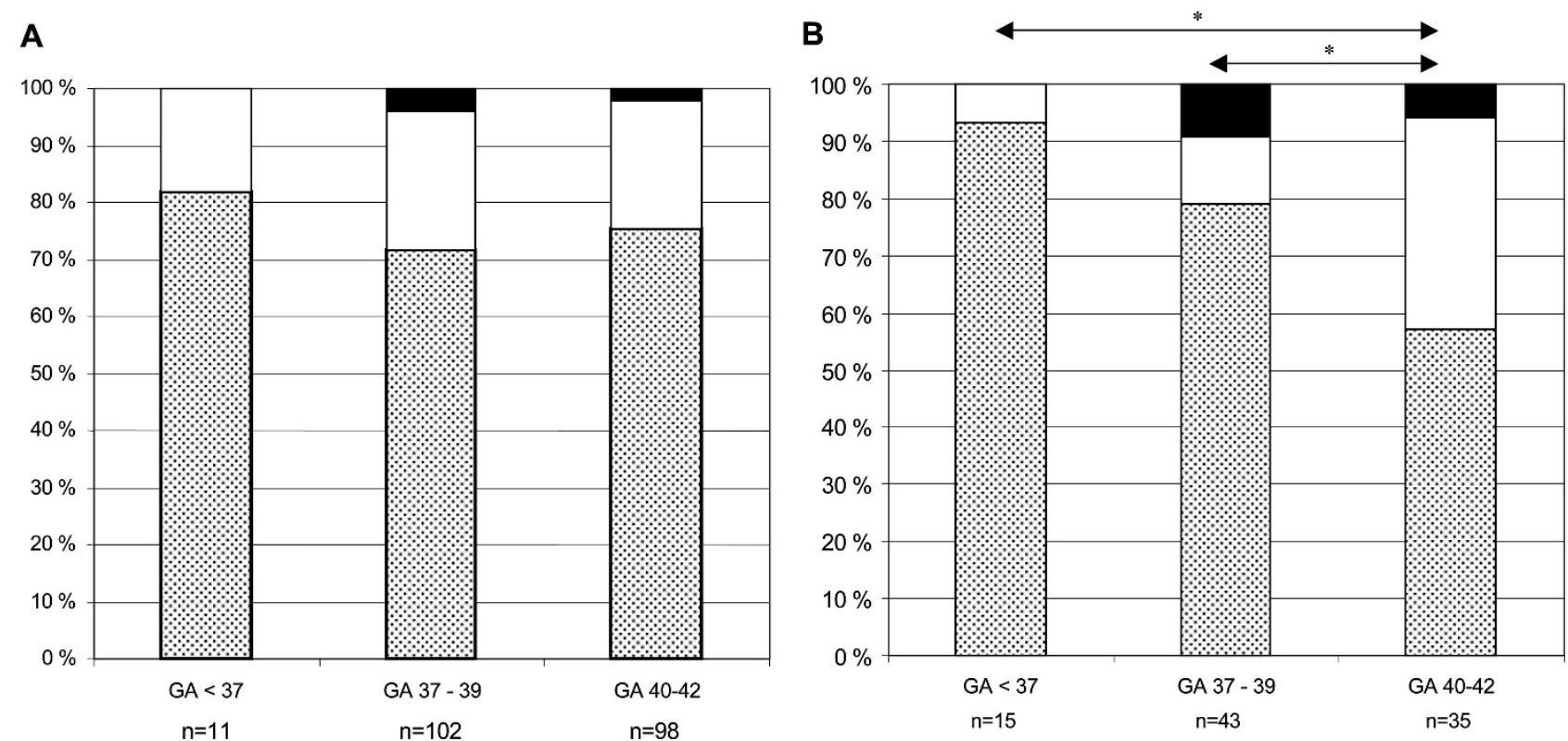

Figure 1. The proportions of boys homozygous for WT-LH (shaded) and V-LH (black) and heterozygous for V-LH (white) according to the gestational age among healthy controls $(A)$ and cryptorchid boys $(B)$. In statistical analyses, boys homozygous for V-LH were combined with boys heterozygous for V-LH. Significantly different proportions $(p<0.05)$ between groups are indicated with an asterisk. 
lower progesterone levels than controls. However, in logistic regression analysis, cryptorchidism had no association with hCG $(p=0.45)$ or progesterone $(p=0.08)$ (Table 1$)$. Instead, LH levels were associated with cryptorchidism. Cryptorchid boys had significantly higher LH levels $(p<0.05$; OR, 1.29; 95\% CI, 1.02-1.63) compared with controls (median, 1.7 IU/L versus $1.3 \mathrm{IU} / \mathrm{L}$, respectively), but this difference was not associated with the V-LH status $(p=0.62)$. In this study, no difference was seen in LH, hCG, or progesterone levels between uni- and bilaterally cryptorchid boys.

\section{DISCUSSION}

This is the first study on the contribution of V-LH status to the development of cryptorchidism. The boys included in this study were recruited prospectively, without any selection bias, so they represent a random population with all degrees of cryptorchidism. Moreover, we performed all the clinical examinations to verify all cryptorchidism diagnoses.

V-LH is a common universal polymorphic variant of normal LH with a very high frequency in the Finnish population. V-LH has been linked to menstrual disorders and subfertility (15-18) but most of the reports about the linkage of V-LH with reproductive failure come from Japan. Male reproductive health has been reported to be good in Finland (19) and in the Finnish studies homozygous V-LH females were fertile (20) with regular menstrual cycles (21). Neither was the proportion of V-LH increased in patients with habitual abortions. In healthy boys, V-LH cause slower pubertal progression but does not affect the onset of puberty (22). The clinical significance of V-LH is still open and in this study we determined whether the occurrence of V-LH is related to testicular descent in the perinatal period.

Cryptorchidism is a significant clinical problem; it is the most common malformation in newborn boys and it is a risk factor for testicular cancer and subfertility in adulthood. Due to the complex etiology of cryptorchidism, a lot of contradictory results have been published. However, a proper hypothalamopituitary-gonadal axis and normal androgen synthesis and action are commonly accepted to be essential for normal testicular descent. hCG is thought to be the main regulator of testicular steroid hormone synthesis in early to mid-pregnancy, but in late pregnancy the role of pituitary gonadotropins seems to become more critical. One proof for this is the increased frequency of cryptorchidism in hypogonadotrophic hypogonadism, despite the fact that hCG production is normal (23). Penile growth is regulated by testosterone, and hypothalamic or pituitary dysfunction does not affect the early phallic differentiation or growth but causes decreased penile growth (micropenis) in the latter half of gestation (24). In this material, cryptorchid boys had higher LH levels, which is in agreement with the theory of insufficient gonadal action in cryptorchidism. However, different LH levels were not associated with the V-LH status.

In this study, the total prevalence of V-LH (26.3\%) corresponds well to the prevalence of V-LH in general Finnish population $(27.7 \%)$. Because V-LH has been presumed to be the less potent form of $\mathrm{LH}$, and insufficient $\mathrm{LH}$ action is related to cryptorchidism, V-LH could be a potent risk factor for cryptorchidism. However, V-LH was as common in cryptorchid as in control group and it did not affect laterality or spontaneous descent of testis after birth. The rate of boys homozygous for V-LH was considerably high $(6.5 \%)$ in the cryptorchid group but no statistically significant difference was found in prevalence of V-LH between the cryptorchid and control group. However, the longer the pregnancy, the higher prevalence of V-LH was found among cryptorchid boys: 6.7\% (GA < 37), 20.9\% (GA 37-39), and 42.9\% (GA 40-42). The continuous increasing trend in the prevalence of $\mathrm{V}-\mathrm{LH}$ was

Table 1. Results (median and quartiles) of human chorionic gonadotropin (hCG), progesterone, and LH measurements in the groups of cryptorchid boys and healthy control boys

\begin{tabular}{|c|c|c|c|}
\hline & Cryptorchid & Control & $\begin{array}{c}p \text { Value* } \\
\text { OR }(95 \% \text { CI })\end{array}$ \\
\hline \multicolumn{4}{|l|}{ HCG (IU/L) } \\
\hline All & $31.0(23.0-54.0)$ & $30.0(18.0-48.0)$ & $\begin{array}{l}0.45 \\
1.74(0.77-3.95) 1 \text { vs } 4 \\
1.54(0.67-3.52) 2 \text { vs } 4 \\
1.87(0.84-4.17) 3 \text { vs } 4\end{array}$ \\
\hline WT- LH & $32.5(23.0-54.0)$ & $29.0(17.0-47.0)$ & \\
\hline V-LH & $29.0(24.0-50.0)$ & $32.0(19.0-49.0)$ & \\
\hline \multicolumn{4}{|c|}{ Progesterone $(\mu \mathrm{mol} / \mathrm{L})$} \\
\hline All & $2.93(2.20-3.69)$ & $3.21(2.28-4.05)$ & $\begin{array}{l}0.08 \\
0.82(0.65-1.02)\end{array}$ \\
\hline WT- LH & $2.92(2.14-3.69)$ & $3.26(2.38-3.94)$ & \\
\hline V-LH & $2.93(2.20-3.69)$ & $2.98(2.12-4.67)$ & \\
\hline \multicolumn{4}{|l|}{ LH (IU/L) } \\
\hline All & $1.66(0.35-2.54)$ & $1.27(0.28-1.95)$ & $\begin{array}{l}0.03 \\
1.29(1.02-1.63)\end{array}$ \\
\hline WT- LH & $1.74(0.73-2.64)$ & $1.32(0.26-1.99)$ & \\
\hline V-LH & $1.40(0.25-2.15)$ & $1.08(0.34-1.88)$ & \\
\hline
\end{tabular}

$p$ Values, odds ratios (OR), and $95 \%$ confidence interval (CI) are used to indicate a statistically significant difference.

*Logistic regression analysis was performed using the model with categorized hCG, LH, and progesterone as independent variables and status as a response variable. Due to nonlinear association with cryptorchidism, the hCG values were divided in four categories using $25 \%$, $50 \%$, and $75 \%$ quartiles as the cut points and group 4 (highest values) was used as the reference group. Statistical analysis was based on hormone measurements of 78 cryptorchid and 165 control boys. 
seen among cases also with more full-term subgroups (limits $39^{+2}$ and $40^{+4}$ are based on $33.3 \%$ percentiles ): $6.7 \%$ (GA $<37$ ), $17.8 \%\left(\right.$ GA $37^{+0}-39^{+1}$ ), 32.0\% (GA 39 $9^{+2}-40^{+4}$ ), and $44.0 \%\left(\mathrm{GA}>40^{+4}\right)$. Variant $\mathrm{LH}$ had no association with $\mathrm{hCG}$ or progesterone levels.

Our results indicate that the V-LH is not critical for normal testicular descent. However, the high prevalence of V-LH among cryptorchid boys born after long pregnancy is in agreement with the supposition that the sufficient pituitary gonadotropin effect is necessary for testicular descent in late pregnancy. We suppose that V-LH is more common in cryptorchid boys in long pregnancies due to its lower biologic efficacy. The longer the pregnancy, the more important role LH may have in relation to that of hCG. In a case of delayed testicular descent, $\mathrm{V}$-LH is not as effective as WT-LH to finish normal testicular descent in late pregnancy. Because testes are usually descended earlier in pregnancy, this weaker efficacy of $\mathrm{V}-\mathrm{LH}$ in late pregnancy does not increase the total risk of cryptorchidism. However, our conclusion that V-LH is very common in cryptorchid boys with long pregnancy should be tested in another population.

It may thus be that the relative importance of pituitary $\mathrm{LH}$, in comparison to $\mathrm{hCG}$, in the stimulation of fetal testicular testosterone production increases with advancing length of gestation. Therefore, as the gestation length increases, the quality of LH secretion becomes important, and V-LH being weaker than WT-LH, is not able to guarantee testicular descent in the overdue boys.

Acknowledgments. The authors thank Mr. Lauri Sillanmäki for assistance with statistical analyses.

\section{REFERENCES}

1. Toppari J, Kaleva M 1999 Maldescendus testis. Horm Res 51:261-269

2. Toppari J, Kaleva M, Virtanen HE 2001 Trends in the incidence of cryptorchidism and hypospadias, and methodological limitations of registry-based data. Hum Reprod Update 7:282-286

3. Gendrel D, Roger M, Job JC 1980 Plasma gonadotropin and testosterone values in infants with cryptorchidism. J Pediatr 97:217-220

4. Job JC, Toublanc JE, Chaussain JL, Gendrel D, Garnier P, Roger M 1988 Endocrine and immunological findings in cryptorchid infants. Horm Res 30:167-172

5. Hadziselimovic F, Girard J, Hocht B, von der Ohe M, Stalder G 1980 Effect of LH-RH treatment on hypothalamo-pituitary-gonadal axis and Leydig cell ultrastructure in cryptorchid boys. Horm Res 13:358-366
6. Pyorala S, Huttunen NP, Uhari M 1995 A review and meta-analysis of hormonal treatment of cryptorchidism. J Clin Endocrinol Metab 80:2795-2799

7. Winter JS, Faiman C, Hobson WC, Prasad AV, Reyes FI 1975 Pituitary-gonadal relations in infancy. I. Patterns of serum gonadotropin concentrations from birth to four years of age in man and chimpanzee. J Clin Endocrinol Metab 40:545-551

8. Winter JS, Hughes IA, Reyes FI, Faiman C 1976 Pituitary-gonadal relations in infancy: 2. Patterns of serum gonadal steroid concentrations in man from birth to two years of age. J Clin Endocrinol Metab 42:679-686

9. Lamminen T, Huhtaniemi I 2001 A common genetic variant of luteinizing hormone; relation to normal and aberrant pituitary-gonadal function. Eur J Pharmacol 414:1-7

10. Nilsson C, Pettersson K, Millar RP, Coerver KA, Matzuk MM, Huhtaniemi IT 1997 Worldwide frequency of a common genetic variant of luteinizing hormone: an international collaborative research. International Collaborative Research Group. Fertil Steril 67:998-1004

11. Nilsson C, Jiang M, Pettersson K, Iitia A, Makela M, Simonsen H, Easteal S, Herrera RJ, Huhtaniemi I 1998 Determination of a common genetic variant of luteinizing hormone using DNA hybridization and immunoassays. Clin Endocrinol (Oxf) 49:369-376

12. Suganuma N, Furui K, Kikkawa F, Tomoda Y, Furuhashi M 1996 Effects of the mutations (Trp8 Arg and Ile15 Thr) in human luteinizing hormone (LH) beta-subunit on LH bioactivity in vitro and in vivo. Endocrinology 137:831-838

13. Pettersson K, Ding YQ, Huhtaniemi I 1992 An immunologically anomalous luteinizing hormone variant in a healthy woman. J Clin Endocrinol Metab 74:164-171

14. Jiang M, Pakarinen $\mathrm{P}$, Zhang FP, El-Hefnawy $\mathrm{T}$, Koskimies $\mathrm{P}$, Pettersson $\mathrm{K}$, Huhtaniemi I 1999 A common polymorphic allele of the human luteinizing hormone beta- subunit gene: additional mutations and differential function of the promoter sequence. Hum Mol Genet 8:2037-2046

15. Furui K, Suganuma N, Tsukahara S, Asada Y, Kikkawa F, Tanaka M, Ozawa T, Tomoda Y 1994 Identification of two point mutations in the gene coding luteinizing hormone (LH) beta-subunit, associated with immunologically anomalous LH variants. J Clin Endocrinol Metab 78:107-113

16. Suganuma N, Furui K, Furuhashi M, Asada Y, Kikkawa F, Tomoda Y. 1995 Screening of the mutations in luteinizing hormone beta-subunit in patients with menstrual disorders. Fertil Steril 63:989-995

17. Takahashi K, Kurioka H, Ozaki T, Kanasaki H, Kohsaka M, Miyazaki K, Karino K 1998 Increased prevalence of luteinizing hormone beta-subunit variant in Japanese infertility patients. Hum Reprod 13:3338-3344

18. Cramer DW, Petterson KS, Barbieri RL, Huhtaniemi IT 2000 Reproductive hormones, cancers, and conditions in relation to a common genetic variant of luteinizing hormone. Hum Reprod 15:2103-2107

19. Toppari J, Larsen JC, Christiansen P, Giwercman A, Grandjean P, Guillette LJ Jr., Jegou B, Jensen TK, Jouannet P, Keiding N, Leffers H, McLachlan JA, Meyer O, Muller J, Rajpert-De Meyts E, Scheike T, Sharpe R, Sumpter J, Skakkebaek NE 1996 Male reproductive health and environmental xenoestrogens. Environ Health Perspect 4:741-803

20. Haavisto AM, Pettersson K, Bergendahl M, Virkamaki A, Huhtaniemi I 1995 Occurrence and biological properties of a common genetic variant of luteinizing hormone. J Clin Endocrinol Metab 80:1257-1263

21. Tulppala M, Huhtaniemi I, Ylikorkala O 1998 Genetic variant of luteinizing hormone in women with a history of recurrent miscarriage. Hum Reprod 13:2699-2702

22. Raivio T, Huhtaniemi I, Anttila R, Siimes MA, Hagenas L, Nilsson C, Pettersson K, Dunkel L 1996 The role of luteinizing hormone-beta gene polymorphism in the onset and progression of puberty in healthy boys. J Clin Endocrinol Metab 81:3278-3282

23. Quinton R, Duke VM, Robertson A, Kirk JM, Matfin G, de Zoysa PA, Azcona C, MacColl GS, Jacobs HS, Conway GS, Besser M, Stanhope RG, Bouloux PM 2001 Idiopathic gonadotrophin deficiency: genetic questions addressed through phenotypic characterization. Clin Endocrinol (Oxf) 55:163-174

24. Feldman KW, Smith DW 1975 Fetal phallic growth and penile standards for newborn male infants. J Pediatr 86:395-398 